\title{
Controle do fluxo principal em autoestradas por meio de veículos cooperativos equipados com controle adaptativo de cruzeiro
}

\author{
Jéssica Aquino Chaves ${ }^{1}$, Rodrigo Castelan Carlson ${ }^{2}$, Eduardo Rauh Müller ${ }^{3}$, Werner Kraus Junior \\ ${ }^{1}$ Programa de Pós-Graduação em Engenharia de Automação e Sistemas, UFSC, Brasil, jessicaaquinochaves@gmail.com \\ 2Programa de Pós-Graduação em Engenharia de Automação e Sistemas, UFSC, Brasil, rodrigo.carlson@ufsc.br \\ 3Programa de Pós-Graduação em Engenharia de Automação e Sistemas, UFSC, Brasil, edurauh@gmail.com \\ ${ }^{4}$ Programa de Pós-Graduação em Engenharia de Automação e Sistemas, UFSC, Brasil, werner.kraus@ufsc.br
}

\section{Recebido:}

16 de março de 2018

Aceito para publicação:

04 de setembro de 2018

Publicado:

4 de novembro de 2018

Editor de área:

Flávio Cunto

\section{Palavras-chaves:}

Controle do Fluxo Principal;

Limites Variáveis de Velocidade;

Veículos cooperativos.

\section{Keywords:}

Mainstream Traffic Flow Control,

Variable Speed Limits,

Cooperative Vehicles.

DOI:10.14295/transportes.v26i3.1629

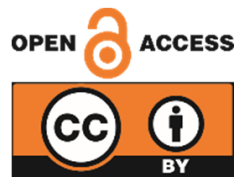

\section{RESUMO}

O Controle do Fluxo Principal (CFP) em autoestradas é um método de controle de tráfego que regula o fluxo de veículos a montante de um gargalo a fim de maximizar o escoamento do fluxo de tráfego na autoestrada. Usando Limites de Velocidade Variáveis (LVV) como atuador do CFP, é analisada a influência de diferentes taxas de penetração de veículos cooperativos no tráfego. Veículos cooperativos foram equipados com Controle Adaptativo de Cruzeiro e recebem como valor de referência o LVV da seção autoestrada em que se encontram. Simulações com o simulador microscópico de tráfego AIMSUN mostraram que o aumento da taxa de penetração contribuiu para o aumento do desempenho. Em cenários cuja taxa de penetração é de 10\%, houve uma melhoria de desempenho de $25 \%$. A presença de mais de $50 \%$ de veículos cooperativos tem um efeito positivo nas condições de tráfego. Porém, é necessária uma estratégia auxiliar para facilitar a inserção dos veículos ao fluxo principal em gargalos da autoestrada ativados por rampas de acesso.

\begin{abstract}
Mainstream Traffic Flow Control (MTFC) on freeways is a traffic control method that aims at regulating vehicle flow upstream from a bottleneck in order to maximize the freeway throughput. Using Variable Speed Limits (VSL) as MTFC actuators, an analysis of the influence of different penetration rates of cooperative vehicles on traffic is made. Cooperative vehicles were equipped with Adaptive Cruise Control (ACC) and receive as a reference value the VSL of the current freeway section. Simulations with the microscopic traffic simulator AIMSUN showed that an increase in penetration rate of cooperative vehicles contributed to performance improvement. Scenarios with a penetration rate of $10 \%$ had a performance improvement of $25 \%$. The presence of more than $50 \%$ cooperative vehicles has a positive effect on traffic conditions. However, an auxiliary control method is necessary to facilitate the merging of vehicles into the mainstream flow in bottlenecks activated by freeway on-ramps.
\end{abstract}

\section{INTRODUÇÃO}

Técnicas de controle ativo do tráfego rodoviário visam melhorar o aproveitamento da infraestrutura viária para que esta opere próxima à capacidade de projeto, algo que raramente ocorre na operação não controlada de autoestradas (Papageorgiou et al., 2003). Neste trabalho, examina-se a técnica de controle que emprega limites variáveis de velocidade (LVV) (Khondaker and Kattan, 2015a) para controle do fluxo principal (CFP) (Carlson et al., 2013) de rodovias considerando a presença de veículos cooperativos.

O CFP busca controlar o fluxo que passa por gargalos viários para maximizá-lo (Carlson et 
al., 2013). No caso do uso de LVV para este controle, explora-se o efeito de redução da capacidade em função da imposição de limites de velocidade menores do que os nominais de projeto. Assim, a aplicação criteriosa de LVVs permite variações dinâmicas da capacidade viária, prevenindo que fluxos muito altos atinjam gargalos e provoquem congestionamentos difíceis de dissolver. Os pontos de aplicação, situados a distâncias adequadas a montante de gargalos, experimentam densidades maiores quando limites menores são aplicados. Parte-se da premissa de que há capacidade viária nos pontos de aplicação tal que esses aumentos de densidade provoquem efeitos menos danosos ao tráfego do que seriam observados no caso de fluxos sem controle atingirem gargalos.

Para serem eficazes, técnicas de controle baseadas em LVV dependem da observância dos limites de velocidade pelos motoristas (Harms e Brookhuis, 2016). As taxas de observância podem ser baixas no caso de trajetos cotidianos (Harms e Brookhuis, 2016) e em rodovias em geral, exceto para seções sob monitoramento por fiscalização eletrônica de velocidade (Soriguera et al., 2015; Riggins et al., 2016). No caso de CFP-LVV por controle realimentado (Carlson et al., 2013; Müller et al., 2015), a baixa observância dos limites de velocidade pode ser compensada pelo controlador que induz a redução do limite de velocidade até que o efeito desejado no fluxo seja atendido ou que um limite inferior seja atingido, caso em que o controlador satura e nada mais pode ser feito.

Os avanços em sistemas de automação e comunicação veicular (VACS - Vehicle Automation and Communication Systems) propiciam nova direção para os sistemas de gerenciamento de tráfego (Roncoli et al., 2015). No caso específico de sistemas baseados em LVV, os limites de velocidade podem ser transmitidos diretamente para o veículo, acelerando o tempo de resposta ao LVV comandado. Além disso, os limites de velocidade podem até mesmo ser impostos, contornando o problema de observância dos LVVs. De fato, várias técnicas que se beneficiam de VACS, de sistemas cooperativos ou de integração veículo-infraestrutura (V2I - Vehicle-to-Infrastructure) foram propostos, tais como Hegyi et al. (2013); Kattan et al. (2015); Khondaker e Kattan (2015b); Roncoli et al. (2015); Davis (2016); Grumert et al. (2015); Müller et al. (2016). Entretanto, desses trabalhos, apenas Müller et al. (2016) adotou a abordagem CFP e nenhum deles considerou veículos equipados com controle adaptativo de cruzeiro (ACC - Adaptive Cruise Control) (Kesting et al., 2007).

Neste artigo, estende-se o trabalho de Müller et al. (2016) ao incorporar veículos cooperativos equipados com ACC, assumindo-se integração veículo-infraestrutura. 0 objetivo é avaliar o desempenho de CFP-LVV na presença de veículos equipados com VACS. São analisadas diferentes taxas de penetração de veículos equipados em situações diversas de densidades de tráfego e de aplicação de sinais de LVV. As simulações são realizadas com o simulador de tráfego Aimsun na mesma autoestrada hipotética modelada por Müller et al. $(2015,2016)$.

\section{REVISÃO DA LITERATURA}

\subsection{Controle do fluxo principal}

Controle do Fluxo Principal (CFP) é uma estratégia de gerenciamento de tráfego que visa maximizar o escoamento dos veículos na via (Carlson et al., 2013). Esta maximização é feita ao reduzir o fluxo de veículos a montante de um gargalo, evitando ou mitigando a formação de congestionamentos e a queda de capacidade no mesmo. A queda de capacidade é um fenômeno identificado quando um congestionamento se forma, fazendo com que a via opere abaixo de sua capacidade nominal. 
De acordo com Papageorgiou et al. (2008), limites de velocidade baixos induzem um fluxo de capacidade mais baixo. Logo, o LVV pode ser utilizado como atuador do CFP. A retenção dos veículos por meio da redução da velocidade cria na região de aplicação do LVV um congestionamento controlado. Essa região, denominada área de aplicação (ver Figura 1), deve ser localizada à uma distância suficientemente a montante do gargalo para que os veículos consigam acelerar e chegar à região do gargalo com a velocidade (crítica) correspondente ao fluxo máximo, permitindo que a via opere em sua capacidade. 0 segmento de via entre a área de aplicação e o gargalo é chamado de área de aceleração.

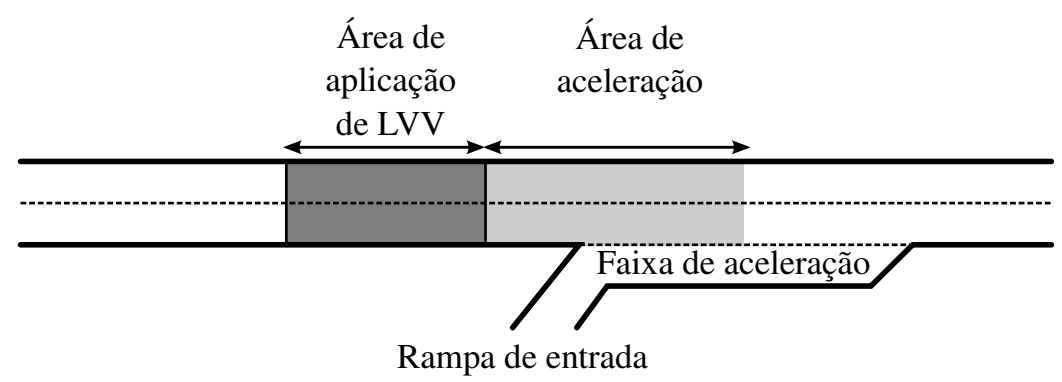

Figura 1: Representação do uso de CFP-LVV em uma autoestrada com rampa de acesso

\subsection{Controle Adaptativo de Cruzeiro - ACC}

Neste trabalho, considera-se a disponibilidade, em diferentes níveis de penetração, de veículos equipados com Controle Adaptativo de Cruzeiro (ACC). O ACC está disponível há anos no mercado automotivo, tendo sido inicialmente desenvolvido para melhorar o conforto e a segurança dos passageiros. Um veículo equipado com essa tecnologia exige da parte do motorista apenas a configuração de uma velocidade desejada e de uma distância ou intervalo temporal (headway) entre o veículo equipado e o veículo à sua frente. Uma vez ativado, o sistema controla a velocidade do veículo para que fique próxima à velocidade desejada. Caso o veículo equipado aproxime-se demais de um veículo à sua frente, por exemplo, por estar mais rápido que ele, o controle passa a manter a distância (ou headway) de segurança definida. Tipicamente, qualquer ação do motorista, como o acionamento do freio, desabilita temporariamente o ACC.

Com o avanço dos VACS, o ACC pode ter um novo papel como um elemento de um sistema de controle hierárquico em que a velocidade desejada não é mais indicada pelo motorista, mas por um sistema de gerenciamento de tráfego que comunica as velocidades desejadas via V2I. Os veículos equipados com ACC tipicamente têm menores tempos de resposta aos comandos de velocidade e a perturbações no tráfego (Shladover, 2012), portanto, potencialmente contribuem para uma homogeneização do fluxo do tráfego (Ntousakis et al., 2015; Shladover, 2012) e para uma redução no tempo total de viagem (Shladover, 2012). Além disso, Ntousakis et al. (2015) avaliaram esse tipo de sistema com diferentes taxas de penetração de veículos equipados com ACC e diferentes headways e constataram, como esperado, que quanto menor o headway entre veículos, maior a capacidade da via.

\section{APLICAÇÃO EM CENÁRIO DE SIMULAÇÃO}

Para estudar o efeito da introdução de veículos equipados com ACC e com equipamentos VACS no desempenho de técnicas de controle do fluxo principal com LVVs, utiliza-se simulação microscópica do tráfego. Nesta seção, apresentam-se os detalhes do ambiente de simulação, dos parâmetros veiculares e de tráfego, e dos cenários considerados. 
As simulações foram realizadas com o microssimulador de tráfego Aimsun 8.1, utilizando sua MicroSDK (Micro Software Development Kit) com a linguagem C++ e sua interface de programação de aplicações (API - Application Programming Interface) com a linguagem Python 2.7. Foram realizadas doze replicações para cada cenário simulado, a fim de obter um intervalo de confiança de 95\% para os resultados obtidos. 0 número de replicações necessárias foi definido por um processo iterativo (Dowling et al., 2004). Inicialmente foram executadas algumas replicações e o desvio padrão obtido dos resultados foi usado para estimar o número de replicações necessárias para o intervalo de confiança desejado. Após a execução das novas replicações, o novo desvio padrão foi calculado e mais uma vez usado para estimar o número de replicações necessárias. 0 processo continuou até que o número estimado de replicações necessárias fosse igual ao daquele realizado.

\subsection{MODELO DA AUTOESTRADA E DEMANDA}

Foi utilizada a mesma autoestrada hipotética de Müller et al. $(2015,2016)$ (Figura 2) composta pela via principal com duas faixas e uma rampa de acesso. A rampa de acesso permite a entrada de veículos numa (terceira) faixa de aceleração de 200 m de extensão, que se inicia 4 km após o início da via. Esse trecho com três faixas é chamado de área de inserção. Ao final da área de inserção, a redução do número de faixas cria um gargalo em potencial. Seis painéis ou sinais de LVV estão dispostos na via. Os Sinais S1 a S4 estão dispostos a montante da área de aplicação a intervalos de $500 \mathrm{~m}$ para possibilitar a redução gradual dos limites de velocidade e, por conseguinte, da velocidade dos veículos que se aproximam da área de aplicação. O Sinal S5 encontrase no início da área de aplicação, que se estende por 300 metros, e o Sinal S6 encontra-se no final da mesma. O Sinal S6 exibe sempre o limite de velocidade de $100 \mathrm{~km} / \mathrm{h}$ a fim de garantir que os veículos possam acelerar e chegar na área do gargalo com velocidade próxima à velocidade crítica, permitindo o fluxo máximo. 0 limite de velocidade exibido em cada sinal e entre sinais tem variação máxima de $20 \mathrm{~km} / \mathrm{h}$ entre períodos de controle.

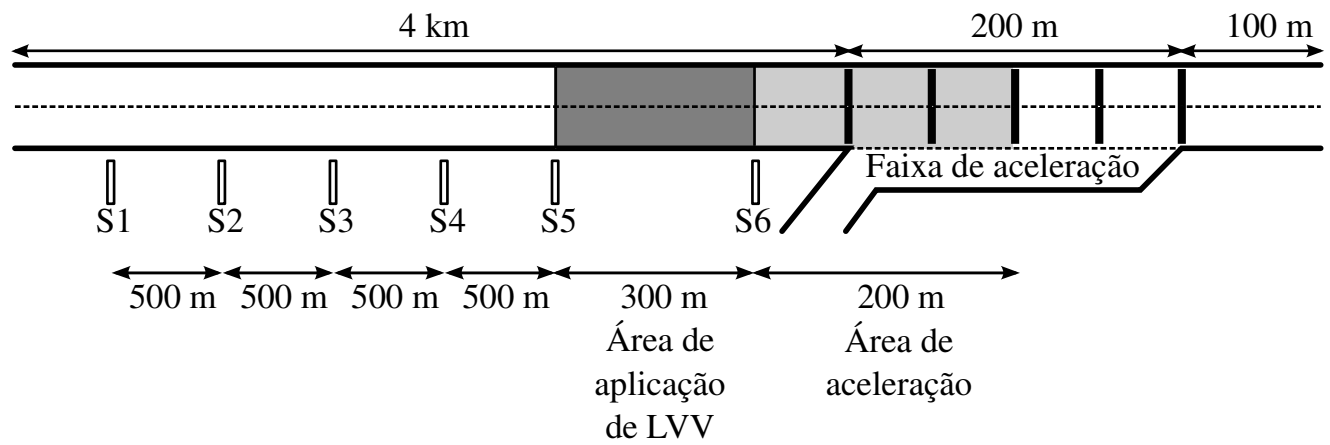

Figura 2: Esquema representativo da autoestrada simulada

A demanda utilizada também foi a mesma de Müller et al. $(2015,2016)$, apresentada na Figura 3 para um período de simulação de 3 horas. A simulação começa com a autoestrada vazia. A demanda é alta o suficiente para criar um congestionamento no cenário sem controle e vai a zero meia hora antes do final da simulação a fim de permitir que em todos os cenários (ver Seção 3.4) todos os veículos consigam deixar a autoestrada até o final da simulação. Isso garante que a quantidade de veículos que cruza a autoestrada seja a mesma em todos os cenários e os tempos totais de viagem possam ser comparados. 


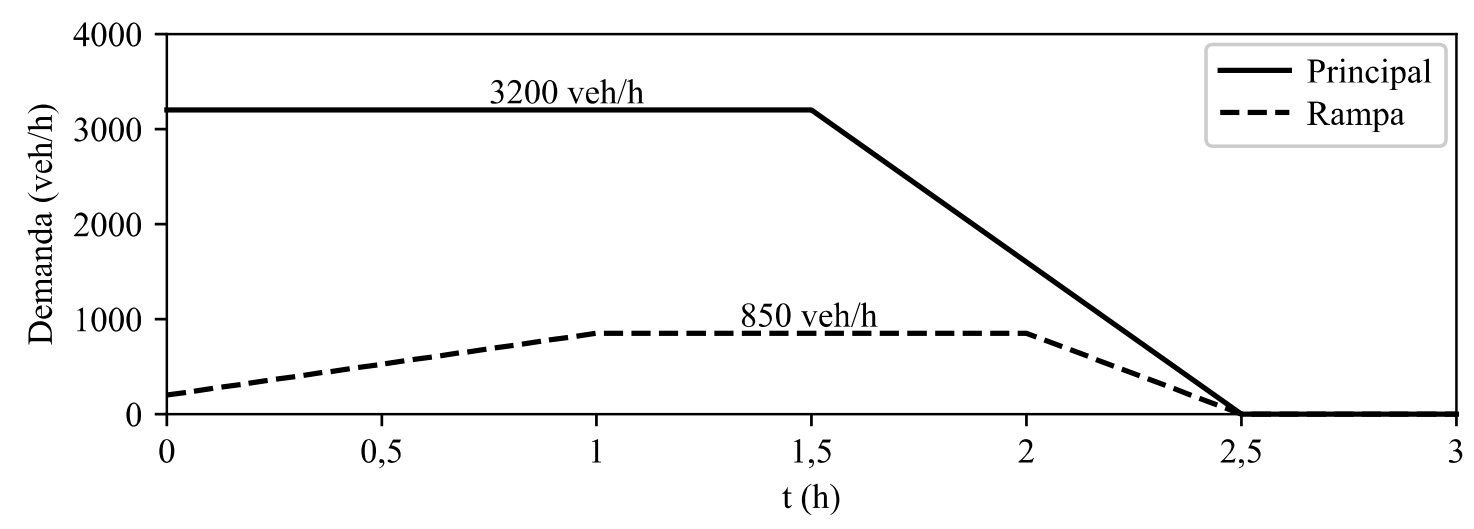

Figura 3: Demanda da via principal e da rampa de acesso

\subsection{Configuração dos veículos}

Neste trabalho foram consideradas duas categorias de veículos leves: veículos convencionais, sem tecnologias que auxiliam o motorista, e cooperativos, no sentido de receberem informações de controle da infraestrutura, que contam com controle adaptativo de cruzeiro.

O movimento longitudinal dos veículos convencionais é regido pelo modelo de car-following de Gipps (1981) como implementado no Aimsun. Os parâmetros dos veículos estão especificados na Tabela $1 \mathrm{com}$ a respectiva variação máxima. Estas variações inserem estocasticidade no sistema e algumas delas representam o papel do fator humano em veículos convencionais. Para os veículos cooperativos, não foi admitida variabilidade para a maioria dos parâmetros.

0 fator aceitação da velocidade é multiplicado pelo limite de velocidade da via para obter a velocidade seguida pelo veículo. Com isso representa-se a não observância dos limites de velocidade pelos motoristas humanos. Neste estudo, considera-se que os motoristas seguem o limite de velocidade com uma diferença de até $10 \%$. Os veículos cooperativos, por sua vez, seguem o limite de velocidade da via precisamente.

0 tempo de reação para os veículos convencionais é de $1 \mathrm{~s}$. Kesting et al. (2007) sugerem a escolha de $0,2 \mathrm{~s}$ para o tempo de reação dos veículos cooperativos, valor que foi o adotado neste trabalho. Não foram considerados ruídos e atrasos no sistema.

Tabela 1: Configuração dos Parâmetros dos Veículos

\begin{tabular}{lll}
\hline Parâmetros & Veículos convencionais & Veículos cooperativos \\
\hline Comprimento $[\mathrm{m}]$ & $4,0 \pm 0,5$ & $4,0 \pm 0,5$ \\
Velocidade máxima $[\mathrm{km} / \mathrm{h}]$ & $100,0 \pm 10,0$ & $100,0 \pm 0,0$ \\
Aceitação da velocidade & $1,0 \pm 0,1$ & $1,0 \pm 0,0$ \\
Tempo de reação [s] & $1,0 \pm 0,0$ & $0,2 \pm 0,0$ \\
Aceleração máxima [m/ $\left.\mathrm{s}^{2}\right]$ & $3,0 \pm 0,1$ & $2,0 \pm 0,0$ \\
Desaceleração máxima $\left[\mathrm{m} / \mathrm{s}^{2}\right]$ & $4,0 \pm 0,25$ & $2,0 \pm 0,0$ \\
\hline
\end{tabular}

A aceleração máxima e a desaceleração máxima no caso de veículos convencionais são parâmetros do modelo de Gipps (1981) e o valor adotado é o valor padrão do simulador. Para a frenagem, uma desaceleração da magnitude da apresentada na Tabela 1 seria aplicada apenas em um caso de "emergência" e raramente ocorre durante a simulação. Já para os veículos cooperativos, a aceleração/desaceleração máxima funciona como um limite para a aceleração calculada pelo ACC. 0 sistema ACC dos veículos cooperativos neste trabalho segue a estrutura de controle proposta por Shladover et al. (2012) e foi configurado um alcance de $200 \mathrm{~m}$ do sensor para detecção de um veículo à frente, calculado de acordo com Wang et al. (2014). 
Além dos parâmetros dos veículos, o modelo de "car-following de duas faixas" do simulador foi configurado de maneira que a diferença máxima de velocidade entre um veículo e veículos próximos trafegando pela faixa imediatamente à direita seja de $20 \mathrm{~km} / \mathrm{h}$, ou de $30 \mathrm{~km} / \mathrm{h}$ no caso de a faixa à direita fazer parte de uma faixa de aceleração.

Finalmente, foi configurado que $80 \%$ dos veículos aceitam ajustar sua velocidade para facilitar a entrada de outros veículos na via principal, exceto no cenário especificado na Seção 0 em que o valor usado foi de $100 \%$.

Estes parâmetros resultaram em uma capacidade nominal da via de $3900 \mathrm{veh} / \mathrm{h}$ e uma queda de capacidade de $22 \%$ no cenário sem controle, similares aos valores obtidos por Müller et al. $(2015,2016)$. 0 próprio simulador foi usado para obter esses valores com o aumento gradativo da demanda até ocorrer a queda de capacidade

\subsection{Sistema de controle}

O sistema de controle opera por meio de um controle realimentado do tipo integral, que regula a ocupação na região do gargalo através de alterações no limite de velocidade na área de aplicação em intervalos de controle de um minuto. A taxa do limite de velocidade $b$, com $0<b_{\min }<$ $b \leq 1$, é a razão entre o limite de velocidade aplicado e o limite de velocidade nominal (ou máximo) da via, em que $b=1$ representa o limite de velocidade máximo adotado pelo controle e $b_{\text {min }}$ é a taxa correspondente ao limite de velocidade mínimo admitido. A taxa $b$, no intervalo de controle $k$, é dada por:

$$
b(k)=b(k-1)+K_{1} \cdot e_{o}(k)
$$

em que $e_{o}(k)$ é o erro da ocupação dado por $e_{o}(k)=\hat{o}-o(k)$, sendo $\hat{o}$ a ocupação de referência (set-point). Esta ocupação de referência é próxima à ocupação crítica, que corresponde ao valor de ocupação referente ao fluxo de capacidade da via. 0 ganho do controlador $K_{\mathrm{I}}$ é escolhido de acordo com o ponto de operação do sistema a partir de um escalonador de ganhos. Isto é necessário devido à não linearidade da relação limite de velocidade e fluxo de capacidade (ver Müller et al., 2015). Uma vez calculado o valor de $b$, o limite de velocidade a ser aplicado pode ser calculado multiplicando $b$ pelo limite de velocidade nominal.

A ocupação $o(k)$, é medida em cinco detectores na região de inserção da via principal (Figura 2), posicionados a uma distância de $50 \mathrm{~m}$ entre si. Esta configuração foi necessária pois a região exata da via em que os veículos tendem a se inserir no fluxo principal varia conforme as condições de tráfego.

Como a proporção de veículos cooperativos interfere no comportamento do sistema, cada cenário teve a ocupação de referência e ganhos do controlador configurados individualmente. Isto é, várias proporções de veículos cooperativos foram simuladas de maneira a atingir a capacidade e provocar congestionamento, o que permite obter a ocupação crítica e, por sua vez, o ganho do controlador.

\subsection{Configuração dos cenários}

Este trabalho compreende a análise de diversos cenários que diferem conforme a composição do tráfego em termos de veículos convencionais e cooperativos e a forma de aplicação do CFP. Os cenários são agrupados em: (i) cenário base; (ii) cenário LVV, (iii) cenários LVV + ACC, (iv) cenários ACC, (v) cenários LVV + ACC (faixa da esquerda), (vi) cenários ACC (faixa da esquerda) e (vii) cenários ACC 100. 
O cenário base envolve apenas veículos convencionais e nenhum tipo de controle. Este cenário é usado como referência para determinar o desempenho relativo dos demais cenários.

0 cenário LVV também envolve apenas veículos convencionais e o controle $b$ da Equação 1 é aplicado por meio de painel de mensagem variável na área de aplicação LVV (S5 na Figura 2), como descrito na Seção 3.3. Esse sinal e os sinais a montante da área de aplicação (S1 a S4 na Figura 2) obedecem às restrições de variação dos limites de velocidade no sinal como descrito na Seção 3.1. O comportamento dos veículos convencionais com respeito aos limites de velocidade é o mesmo para todos os cenários com LVV.

Os cenários LVV + ACC e ACC, envolvem veículos convencionais e cooperativos. No primeiro conjunto de cenários o controle $b$ da Equação 1 é aplicado tanto por meio de painéis de mensagem variável como por mensagens enviadas ao ACC dos veículos cooperativos, enquanto no segundo conjunto não há painéis de mensagem variável e o controle atinge apenas os veículos cooperativos. Para os dois casos são simuladas combinações com várias taxas de penetração de veículos cooperativos variando de 10 a $100 \%$ em intervalos de $10 \%$.

As simulações envolvendo ACC são repetidas para quatro valores diferentes de headway: 0,8 $\mathrm{s}, 1,0 \mathrm{~s}, 1,4 \mathrm{~s}, 1,8 \mathrm{~s}$. Os valores de 1,4 s e 1,8 s foram escolhidos pois são atingíveis com a tecnologia de ACC (Lin et. al, 2019), enquanto o valor de 1,0 s foi escolhido por corresponder ao estado da arte (Kayacan, 2017). Já o valor de 0,8 s possibilita avaliar o sistema para o caso de um desenvolvimento tecnológico que permita reduzir ainda mais o valor de headway, embora este valor já seja viável por meio de outras tecnologias, como o controle adaptativo de cruzeiro cooperativo (Kayacan, 2017). Notar que as variações de headway são empregadas apenas para os veículos equipados com ACC. Os veículos convencionais continuam operando com os headways resultantes do modelo de seguimento veicular de Gipps.

Como neste trabalho não foram abordadas técnicas auxiliares de inserção de veículos, como as estudadas por Scarinci e Heydecker (2014), duas configurações adicionais do simulador foram empregadas com a intenção de se avaliar se facilitar a entrada dos veículos da rampa na via principal melhora o desempenho quando veículos cooperativos estão presentes. A primeira configuração faz com que os veículos cooperativos se mantenham preferencialmente na faixa da esquerda, o que facilita a entrada dos veículos oriundos da rampa na faixa da direita. Essa configuração foi utilizada nos cenários LVV + ACC (faixa da esquerda) e ACC (faixa da esquerda). A segunda configuração aumenta de 80 para $100 \%$ a taxa de veículos que aceita ajustar sua velocidade para facilitar a inserção de um veículo à sua frente e foi aplicada apenas para o caso de controle via ACC resultando nos cenários ACC 100.

\section{RESULTADOS}

Os resultados das simulações apresentados para cada cenário nesta seção correspondem à média das doze replicações daquele cenário. 0 indicador de desempenho analisado foi o tempo total de viagem (TTV). O TTV [h] representa a soma do tempo gasto para cruzar a autoestrada por todos os veículos, somada ao tempo de espera em fila virtual pelos veículos que não conseguiram entrar imediatamente na autoestrada. Assim, para uma mesma demanda e em condições que os cenários podem ser comparados como neste trabalho, um menor TTV indica melhor desempenho.

\subsection{Análise da taxa de penetração e headway}

A Figura 4 mostra os valores de TTV para os cenários LVV + ACC para as diferentes taxas de 
penetração de veículos cooperativos e para os quatro headways considerados. As linhas horizontais, contínua e tracejada, representam os cenários apenas com veículos convencionais sem controle e com CFP-LVV, respectivamente. 0 TTV do cenário sem controle é de 996 h, e do cenário com controle LVV, de $752 \mathrm{~h}$, uma melhoria de 24,4\% do último em relação ao primeiro.

Todos os cenários com veículos cooperativos apresentados na Figura 4, exceto o cenário com headway de 1,8 s e taxa de penetração de $100 \%$, têm melhor desempenho que o cenário sem controle. A maioria dos cenários também apresenta desempenho tão bom ou melhor que o cenário LVV. Note que mesmo com uma taxa de penetração de apenas $10 \%$, ocorre uma melhoria de $7 \%$ no cenário com headway de 0,8 s e uma melhoria de cerca de $24 \%$ nos cenários com headways de 1,0 s a 1,8 s em relação ao cenário sem controle.

Na faixa de 30 a $60 \%$ de taxa de penetração, todos os cenários apresentam uma melhora acentuada em relação ao cenário sem controle, tendo um desempenho ainda melhor do que no caso em que apenas CFP-LVV é aplicado. É notório o melhor desempenho dos cenários com headways de 0,8 s e 1,0 s, como era de se esperar, uma vez que esses valores de headway proporcionam maior capacidade para a via. Na faixa de 60 a $90 \%$ de taxa de penetração a melhora é bastante discreta ou estagnada. Para valores maiores de headway o desempenho tende a regredir, a partir de uma taxa de $80 \%$.

Para taxas de penetração altas, isto é, acima de 60 ou 70\%, a alta proporção de veículos cooperativos combinada à alta demanda passa a dificultar a inserção de veículos oriundos da rampa na via principal, devido ao espaçamento constantemente baixo entre os veículos e à baixa variabilidade deste espaçamento. Como consequência, o CFP-LVV não é ativado, pois a ocupação permanece baixa na via principal, ao passo que filas começam a ser formadas na rampa de entrada. A despeito disso, o desempenho do sistema só é afetado negativamente a partir de uma taxa de penetração de 80 a 90\%, pois a capacidade auferida pelo uso de ACC e as brechas ainda disponíveis até então são suficientes para que o TTV seja mantido baixo. Com $100 \%$ dos veículos cooperativos, as filas aumentam significativamente, particularmente para os headways de 1,4 s e 1,8 s, de tal sorte que muitos veículos permanecem na fila (virtual) e só são liberados quando a demanda é reduzida a zero próximo ao final da simulação.

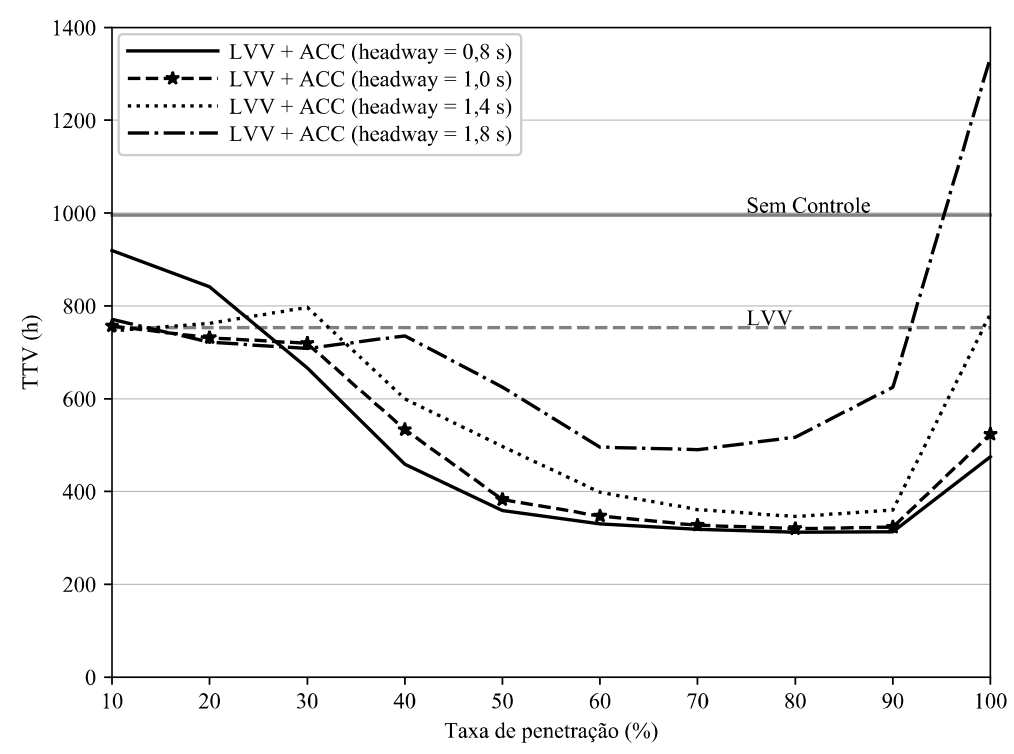

Figura 4. Tempo total de viagem para os cenários com controle LVV + ACC em função da taxa de penetração de veículos cooperativos equipados com ACC para diferentes valores de headway 


\subsection{Análise de cenários selecionados}

Os resultados da seção anterior sugerem que para altas taxas de penetração de veículos cooperativos e alta demanda, os veículos oriundos da rampa podem ter dificuldade de se inserir na via principal. De fato, segundo Ntousakis et al. (2016), para garantir que os veículos da rampa de acesso consigam se inserir na via principal, os veículos da via principal devem desacelerar quando chegam na área de inserção, para criar espaçamentos apropriados. A implementação de uma estratégia de inserção cooperativa ou facilitada (Scarinci e Heydecker, 2014) não foi realizada neste trabalho. Porém, foram feitos alguns testes que tiraram proveito de recursos do simulador que favorecem ou facilitam a inserção de veículos.

As simulações desta seção foram realizadas, para as diferentes taxas de penetração e headways. Como os resultados foram semelhantes para todos os headways analisados, serão mostrados aqui apenas os cenários com o headway de 1,4 s.

Na Figura 5, a linha contínua representa o TTV do cenário com controle LVV + ACC para diferentes taxas de penetração com headway 1,4 s. Para esses cenários, foi feita a análise de como o comportamento do sistema é afetado quando os veículos cooperativos se mantêm na faixa da esquerda. Para taxas de penetração abaixo de 30\%, houve uma piora do desempenho do sistema. Isto acontece pois houve um aumento do número de troca de faixas, o que gerou um aumento de perturbações que, por sua vez, afetaram o desempenho. Com o aumento do número de veículos cooperativos, estas perturbações puderam ser melhor acomodadas no sistema.

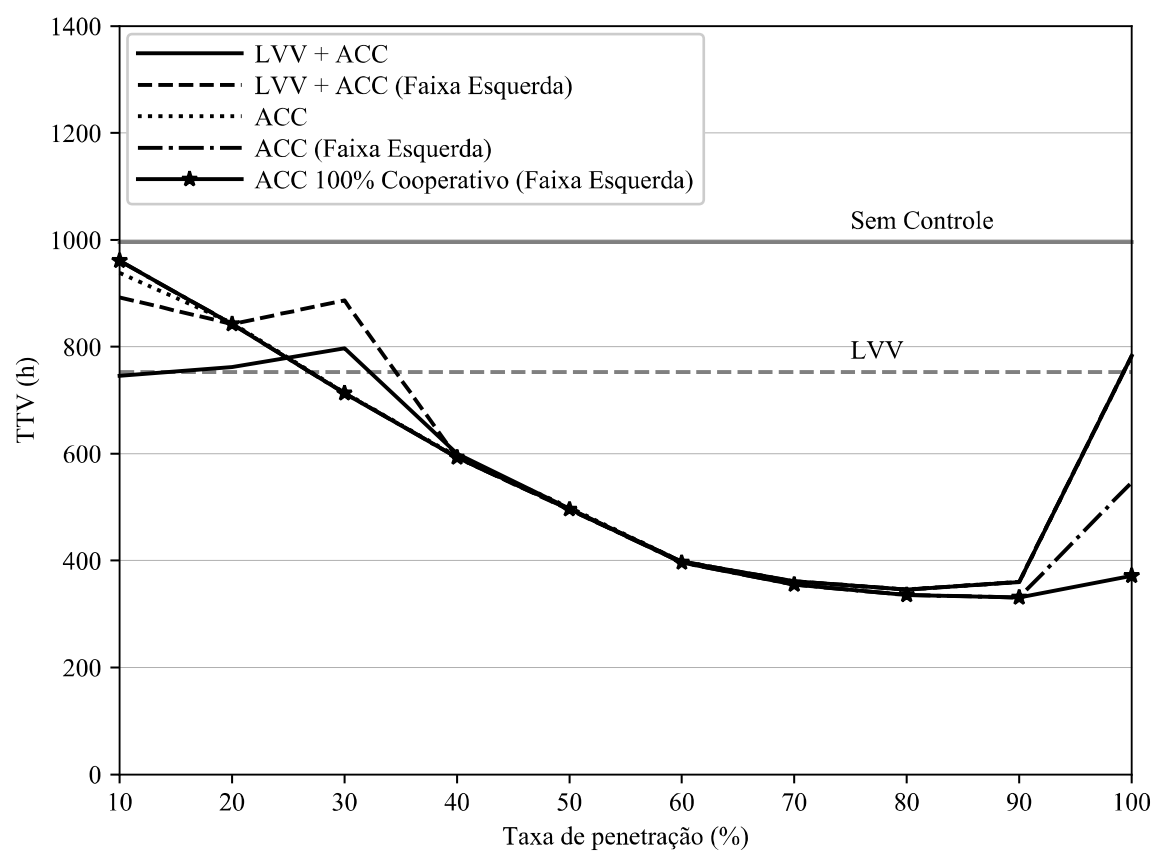

Figura 5. Tempo total de viagem em função das taxas de penetração de veículos cooperativos equipados com ACC e com headway de 1,4 s, para diferentes estratégias de controle e de facilitação de inserção

Também foram avaliados cenários sem o controle CFP-LVV, para verificar qual a contribuição apenas dos veículos cooperativos na melhora do desempenho do sistema. Os cenários que contêm somente veículos equipados com ACC apresentaram melhor desempenho quando a cooperação para a troca de faixas é de 100\%, confirmando a importância de técnicas de inserção cooperativa ou facilitada. 


\section{CONCLUSÃO}

Este trabalho propõe uma extensão do trabalho de Müller et al. (2016), introduzindo veículos cooperativos equipados com controle adaptativo de cruzeiro no sistema. Estes veículos recebem a informação do limite de velocidade da via, auxiliando o controle do fluxo principal por meio de limites variáveis de velocidade a regular o fluxo. Simulações foram realizadas para diferentes taxas de penetração de veículos cooperativos, assim como diferentes headways. Os resultados mostraram que a escolha da taxa de penetração e do headway têm um efeito direto no tempo total de viagem. Mesmo os cenários com baixa taxa de penetração apresentaram um melhor desempenho quando comparados aos cenários sem controle ou somente com o controle do fluxo principal por limites variáveis de velocidade. Para taxas de penetração acima de 30 \%, quanto menor o headway, menor o tempo total de viagem.

Apesar de não terem sido avaliadas estratégias auxiliares de inserção dos veículos da rampa à via principal, é possível notar que a implementação de mudanças no comportamento dos veículos para facilitar a inserção contribui para a melhoria do desempenho do sistema.

\section{AGRADECIMENTOS}

Os autores agradecem o apoio da CAPES e do CNPq e aos revisores anônimos.

\section{REFERÊNCIAS}

Carlson, R. C.; I. Papamichail e M. Papageorgiou (2013) Mainstream traffic flow control on freeways using variable speed limits. Transportes, v. 21, n. 3, p. 56-65. DOI: 10.4237/transportes.v21i3.694

Davis, L. (2016) Improving traffic flow at a 2-to-1 lane reduction with wirelessly connected, adaptive cruise control vehicles. Physica A: Statistical Mechanics and its Applications, v. 451, p. 320-332. D0I: 10.1016/j.physa.2016.01.093

Dowling, R.; A. Skabardonis e V. Alexiadis (2004) Traffic analysis toolbox volume III: guidelines for applying traffic microsimulation modeling software, Publication No. FHWA-HRT-04-040, U.S. Department of Transportation, Federal Highway Administration.

Kayacan, E. (2017) Multiobjective Ho Control for String Stability of Cooperative Adaptive Cruise Control Systems. IEEE Transactions on Intelligent Vehicles, v. 2, n. 1, p. 52-61. D0I: 10.1109/TIV.2017.2708607

Gipps, P. G. (1981) A behavioural car-following model for computer simulation. Transportation Research Part B: Methodological, v. 15, n. 2, p. 105-111. DOI: 10.1016/0191-2615(81)90037-0

Grumert, E.; X. Ma e A. Tapani (2015) Analysis of a cooperative variable speed limit system using microscopic traffic simulation. Transportation Research Part C: Emerging Technologies, v. 52, p. 173-186. D0I: 10.1016/j.trc.2014.11.004

Harms, I. M. e K. A. Brookhuis (2016) Dynamic traffic management on a familiar road: failing to detect changes in variable speed limits. Transportation Research Part F: Traffic Psychology and Behaviour, v. 38, p. 37-46. DOI: 10.1016/j.trf.2016.01.005

Hegyi, A.; B. Netten; M. Wang; W. Schakel; T. Schreiter; Y. Yuan; B. van Arem e T. Alkim (2013) A cooperative system based variable speed limit control algorithm against jam waves - an extension of the SPECIALIST algorithm. The 16th International IEEE Conference on Intelligent Transportation Systems, p. 973-978. DOI: 10.1109/ITSC.2013.6728358

Iordanidou, G. R.; C. Roncoli; I. Papamichail e M. Papageorgiou (2014) Feedback-based mainstream traffic flow control for multiple bottlenecks on motorways. IEEE Transactions on Intelligent Transportation Systems, v. 16, n. 2, p. 610-621. DOI: 10.1109/TITS.2014.2331985

Kesting, A; M. Treiber; M. Schönhof e D. Helbing (2007) Extending adaptive cruise control to adaptive driving strategies. Transportation Research Record: Journal of the Transportation Research Board, n. 2000, p. 16-27. DOI: 10.3141/2000-03

Kattan, L.; B. Khondaker; O. Derushkina e E. Poosarla (2015) A probe-based variable speed limit system. Journal of Intelligent Transportation Systems, v. 19, n. 4, p. 339-354. DOI: 10.1080/15472450.2014.936294

Khondaker, B. e L. Kattan (2015a) Variable speed limit: an overview. Transportation Letters, v. 7, n. 5, p. 264-278. DOI: $10.1179 / 1942787514$ Y.0000000053

Khondaker, B. e L. Kattan (2015b) Variable speed limit: a microscopic analysis in a connected vehicle environment. Transportation Research Part C: Emerging Technologies, v. 58, p. 146-159. DOI: 10.1016/j.trc.2015.07.014

Lin, T.-W.; S.-L. Hwang,e P. A. Green (2009) Effects of time-gap settings of adaptive cruise control (ACC) on driving performance and subjective acceptance in a bus driving simulator. Safety Science, v. 47, n. 5, p. 620-625. DOI: 10.1016/j.ssci.2008.08.004

Müller, E. R.; R. C. Carlson; W. Kraus e M. Papageorgiou (2015) Microsimulation analysis of practical aspects of traffic control with variable speed limits. IEEE Transactions on Intelligent Transportation Systems, v. 16, n. 1, p. 512-523. DOI: 10.1109/TITS.2014.2374167 
Müller, E. R.; R. C. Carlson e W. Kraus (2016) Cooperative mainstream traffic flow control on freeways. IFAC-PapersOnLine. v. 49, n. 32, p. 89-94. DOI: 10.1016/j.ifacol.2016.12.195

Ntousakis, I. A.; I. K. Nikolos e M. Papageorgiou (2015) On microscopic modelling of adaptive cruise control systems. Transportation Research Procedia, v. 6, p. 111-127. DOI: 10.1016/j.trpro.2015.03.010

Papageorgiou, M.; C. Diakaki, V. Dinopoulou, A. Kotsialos e Wang, Y. (2003). Review of road traffic control strategies. Proceedings of the IEEE, 91(12), 2043-2067. DOI: 10.1109/JPROC.2003.819610

Papageorgiou, M.; E. Kosmatopoulos e I. Papamichail (2008) Effects of variable speed limits on motorway traffic flow. Transportation Research Record: Journal of the Transportation Research Board, n. 2047, v. 37-48. DOI: 10.3141/2047-05

Riggins, G.; R. Bertini; W. Ackaah e M. Margreiter (2016). Evaluation of driver compliance to displayed variable advisory speed limit systems: comparison between Germany and the U.S. Transportation Research Procedia, v. 15, p. 640-651. DOI: 10.1016/j.trpro.2016.06.054

Roncoli, C.; M. Papageorgiou e I. Papamichail (2015) Traffic flow optimisation in presence of vehicle automation and communication systems - Part II: optimal control for multi-lane motorways. Transportation Research Part C: Emerging Technologies, v. 57, p. 260-275. DOI: 10.1016/j.trc.2015.05.011

Scarinci, R. e B. Heydecker (2014) Control concepts for facilitating motorway on-ramp merging using intelligent vehicles. Transport Reviews, v. 34, n. 6, p. 775-797. DOI: 10.1080/01441647.2014.983210

Shladover, S.; D. Su e X. Lu (2012) Impacts of cooperative adaptive cruise control on freeway traffic flow. Transportation Research Record: Journal of the Transportation Research Board, n. 2324, p. 63-70. DOI: 10.3141/2324-08

Soriguera, F.; I. Martínez-Josemaría e M. Menéndez (2015) Experimenting with dynamic speed limits on freeways. TRB 94th Annual Meeting Compendium of Papers, Washington, D.C., USA.

Wang, M.; W. Daamen; S. P. Hoogendoom e B. van Arem (2014) Rolling horizon control framework for driver assistance systems. Part I: Mathematical formulation and non-cooperative systems. Transportation Research Part C: Emerging Technologies, v. 40, p. 290-311. DOI: 10.1016/j.trc.2013.11.023 